ARTICLE OPEN

\title{
Quantum nonlocality without entanglement: explicit dependence on prior probabilities of nonorthogonal mirror-symmetric states
}

\author{
Donghoon $\mathrm{Ha} \mathbb{D}^{1,2 \otimes}$ and Younghun Kwon $\mathbb{D}^{2 \otimes}$
}

In the case of a multi-party system, through local operations and classical communication (LOCC), each party may not perform perfect discrimination of quantum states that are separable and orthogonal. This property of quantum ensemble is called "nonlocality without entanglement" since each local party has a limit to full information of given quantum states. When this property is extended to the case of minimum-error discrimination, one can see that it is revealed when a nonlocal measurement provides more information about the unentangled states than LOCC does. One may infer the fact that the property depends on quantum states composing the quantum ensemble. However, an essential but unsettled question about the property is whether an explicit dependence on prior probabilities in terms of minimum-error discrimination could be shown in nonlocality without entanglement. In a simple term, one can ask whether different quantum ensembles made of the same separable quantum states could exhibit explicitly different behavior of the nonlocality. We answer this question in the positive, and we furthermore provide the explicit functional dependence of guessing probability on prior probabilities for the mirror-symmetric ensemble.

npj Quantum Information (2021)7:81; https://doi.org/10.1038/s41534-021-00415-0

\section{INTRODUCTION}

In 1991, Peres and Wootters proposed a seminal problem ${ }^{1}$ : What is the difference between global and local measurements on quantum states prepared at different locations? The question naturally leads to an investigation into the role of local operations and classical communication (LOCC), which is necessary for characterizing entanglement ${ }^{2}$ and the nonlocality of quantum information $^{3-15}$. Because the global measurement is to measure quantum states at different locations as a whole, it can be entangled or not. When it is not entangled, it is called a separable measurement (SEP). In other words, SEP is a measurement consisting of separable operators. A local measurement is to measure locally quantum states at different locations. A local measurement is performed at one location, and the result is sent to the other location to perform a suitable measurement. And LOCC can be finite or infinite ${ }^{16}$. Every LOCC can be realized through SEP, but the inverse does not hold ${ }^{3}$.

To understand the role of LOCC, Peres and Wootters considered the minimum-error discrimination $(M D)^{17-20}$ of double trine ensemble and showed that there is a difference between a global measurement and finite rounds of local measurements on the quantum ensemble ${ }^{1}$. Also, Bennett et al. ${ }^{3}$ showed that nine orthogonal product states could provide "nonlocality without entanglement" (NLWE) because it is impossible to distinguish the nine states by asymptotic $\operatorname{LOCC}^{21,22}$ even though they can be distinguished globally. One should note that the nonlocality does not mean the nonlocality of quantum state, but the nonlocality of quantum ensemble composing the quantum state. Their investigation provides a motivation by which researchers have performed on discrimination of multipartite states under restricted quantum operations of LOCC or SEP S-16,21-26. $^{4}$.
Recently, Chitambar and Hsieh ${ }^{15}$ showed that in terms of MD, NLWE occurs in double trine ensemble ${ }^{1}$, which consists of three symmetric $2 \otimes 2$ product states with identical prior probability. More explicitly, it means that in MD of double trine ensemble the guessing probability of globally optimal measurement is larger than that of asymptotic LOCC ( $\overline{\mathrm{LOCC}})$. The result shows a specific quantum ensemble revealing NLWE when they are shared at different locations, but it cannot tell whether NLWE depends on the manner of forming a quantum ensemble. Then, the following three questions naturally appear. The first one is whether there exists a nonorthogonal state ensemble revealing NLWE as double trine ensemble shows NLWE. The second one is whether one can show NLWE of nonorthogonal state ensemble by appealing SEP, which one can handle more easily than $\overline{\mathrm{LOCC}}$. The last and most important question is whether an explicit dependence on prior probabilities in terms of MD can be shown in NLWE. In other words, one can ask whether there exist nonorthogonal states whose NLWE depends on nonzero prior probabilities. To the best of the authors' knowledge, an answer to this question has not yet been offered.

In this work, we provide three nonorthogonal product states in a two-qubit system where the occurrence of NLWE depends on nonzero prior probabilities. The ensemble's quantum states do not carry a cyclic symmetry as the three states of the double trine ensemble do. However, they possess a mirror symmetry. Using the three quantum states with two different parametrizations, we show that there are the following two instances for nonzero prior probabilities. The one is the case where global minimum-error measurement can be performed only by finite-round LOCC. The other one is that SEP cannot achieve global minimum-error measurement. In the former case, NLWE does not occur, but in the latter case, NLWE does occur. Therefore, our results provide answers to the three questions mentioned previously.

${ }^{1}$ Department of Applied Mathematics and Institute of Natural Sciences, Kyung Hee University, Yongin, Republic of Korea. ${ }^{2}$ Department of Applied Physics, Center for Bionano Intelligence Education and Research, Hanyang University, Ansan, Republic of Korea. ${ }^{凶}$ email: mslab.h@gmail.com; yyhkwon@hanyang.ac.kr 


\section{RESULTS}

The difficulty in understanding the explicit dependence of prior probability in NLWE is due to the fact that a general method to obtain an optimal measurement for three quantum states is not known yet. Therefore, one should consider a suitable quantum ensemble where one can find its optimal measurement and further can discuss the optimality of local measurement.

\section{Case where different quantum states are shared at different locations}

Now, we begin our investigation by considering the case where different quantum states are shared at different locations. The quantum ensemble is given as $\mathcal{E}=\left\{q_{i}, \rho_{i}\right\}_{i=0}^{2}$, and consists of twoqubit product states $\rho_{i}=\left|\Psi_{i}\right\rangle\left\langle\Psi_{i}\right|$ with prior probabilities $q_{i}$. It satisfies the following relation:

$\frac{q_{0}}{q_{1}}=\frac{q_{0}}{q_{2}}=r$ for some $r \in(0, \infty)$.

The ratio $r$ is the only variable indicating the change of prior probabilities. The two-qubit product states $\left|\Psi_{i}\right\rangle\left\langle\Psi_{i}\right|$, which we consider, are as follows (the ranges of $\theta_{0}$ and $\theta_{1}$ become $0<\theta_{0}<\frac{\pi}{4}$ and $\left.0<\theta_{1}<\frac{\pi}{2}-\theta_{0}\right)$ :

$$
\begin{aligned}
\left|\Psi_{0}\right\rangle & =|00\rangle, \\
\left|\Psi_{1}\right\rangle & =\left|A_{+}\right\rangle \otimes\left|B_{+}\right\rangle \\
& =\sqrt{\frac{\sin 2 \theta_{0}}{\sin 2 \theta_{0}+\sin 2 \theta_{1}}}\left|\Phi_{\theta_{1}}^{+}\right\rangle+\sqrt{\frac{\sin 2 \theta_{1}}{\sin 2 \theta_{0}+\sin 2 \theta_{1}}}\left|\Psi_{\theta_{0}}^{+}\right\rangle, \\
\left|\Psi_{2}\right\rangle & =\left|A_{-}\right\rangle \otimes\left|B_{-}\right\rangle \\
& =\sqrt{\frac{\sin 2 \theta_{0}}{\sin 2 \theta_{0}+\sin 2 \theta_{1}}}\left|\Phi_{\theta_{1}}^{+}\right\rangle-\sqrt{\frac{\sin 2 \theta_{1}}{\sin 2 \theta_{0}+\sin 2 \theta_{1}}}\left|\Psi_{\theta_{0}}^{+}\right\rangle,
\end{aligned}
$$

where

$$
\begin{aligned}
& \left|A_{ \pm}\right\rangle=\frac{1}{\sqrt{1+\tan \theta_{0} \tan \theta_{1}}}\left(|0\rangle \pm \sqrt{\tan \theta_{0} \tan \theta_{1}}|1\rangle\right), \\
& \left|B_{ \pm}\right\rangle=\frac{1}{\sqrt{\tan \theta_{0}+\tan \theta_{1}}}\left(\sqrt{\tan \theta_{0}}|0\rangle \pm \sqrt{\tan \theta_{1}}|1\rangle\right),
\end{aligned}
$$

and

$$
\begin{aligned}
& \left|\Phi_{\theta}^{+}\right\rangle=\cos \theta|00\rangle+\sin \theta|11\rangle,\left|\Phi_{\theta}^{-}\right\rangle=\sin \theta|00\rangle-\cos \theta|11\rangle, \\
& \left|\Psi_{\theta}^{+}\right\rangle=\cos \theta|01\rangle+\sin \theta|10\rangle,\left|\Psi_{\theta}^{-}\right\rangle=\sin \theta|01\rangle-\cos \theta|10\rangle .
\end{aligned}
$$

To understand NLWE for this quantum ensemble, we should compare $P_{\text {guess }}^{\mathrm{GLOBA}}$, the guessing probability when the global measurement is used, with $P_{\text {guess }}^{\overline{\mathrm{LOCC}}}$, when only local measurements are allowed. The optimal positive operator-valued measure (POVM) $\left\{\left|\Phi_{i}\right\rangle\left\langle\Phi_{i}\right|\right\}_{i=0}^{2}$ for $\mathcal{E}$ can be described in threedimensional complex Hilbert space generated by $\left\{\left|\Psi_{i}\right\rangle\right\}_{i=0}^{2}$ (see "Methods"):

$$
\begin{aligned}
& \left|\Phi_{0}\right\rangle=\sin X|00\rangle-\cos X|11\rangle, \\
& \left|\Phi_{1}\right\rangle=\frac{1}{\sqrt{2}}(\cos X|00\rangle+\sin X|11\rangle)+\frac{1}{\sqrt{2}}\left|\Psi_{\theta_{0}}^{+}\right\rangle, \\
& \left|\Phi_{2}\right\rangle=\frac{1}{\sqrt{2}}(\cos X|00\rangle+\sin X|11\rangle)-\frac{1}{\sqrt{2}}\left|\Psi_{\theta_{0}}^{+}\right\rangle,
\end{aligned}
$$

where the optimal measurement factor $x \in\left(0, \frac{\pi}{2}\right)$ satisfies the following relation:

$$
r=\frac{\sin 2 \theta_{0} \sin 2\left(x-\theta_{1}\right)+2 \sqrt{\sin 2 \theta_{0} \sin 2 \theta_{1}} \sin \left(x-\theta_{1}\right)}{\sin 2 x\left(\sin 2 \theta_{0}+\sin 2 \theta_{1}\right)} \text {. }
$$

The global guessing probability $P_{\text {guess }}^{\mathrm{GLOBA}}$ of the quantum ensemble $\mathcal{E}$ can be obtained by applying the optimal global measurement. To figure out NLWE, one needs to check whether the global minimum-error probability can be obtained by the finite-round LOCC or asymptotic LOCC ${ }^{21,22}$. It is known that the global minimum-error probability can be attained by finite-round (asymptotic) LOCC if and only if the optimal POVM $\left\{\left|\Phi_{i}\right\rangle\left\langle\Phi_{i}\right|\right\}_{i=0}^{2}$ can be perfectly discriminated using the finite-round (asymptotic)
LOCC (see "Methods"). Here, in understanding this problem, one can recall a result obtained by Walgate and $\mathrm{Hardy}^{5}$. It tells that in a two-qubit system, three pure orthogonal states can be perfectly discriminated by a finite-round LOCC if and only if at least two of them are product states. It means that $\left\{\left|\Phi_{i}\right\rangle\left\langle\Phi_{i}\right|\right\}_{i=0}^{2}$ can be perfectly discriminated by a finite-round LOCC if and only if at least two of $\left\{\left|\Phi_{i}\right\rangle\left\langle\Phi_{i}\right|\right\}_{i=0}^{2}$ are product states. Therefore, the possibility of NLWE depends on whether $\left\{\left|\Phi_{i}\right\rangle\left\langle\Phi_{i}\right|\right\}_{i=0}^{2}$ is entangled or not. By evaluating concurrence ${ }^{27}$, one can see that two of the $\left\{\left|\Phi_{i}\right\rangle\left\langle\Phi_{i}\right|\right\}_{i=0}^{2}$ become unentangled if and only if $\sin 2 X=\sin 2 \theta_{0}$ (i.e., $X=\theta_{0}$ or $\frac{\pi}{2}-\theta_{0}$ ). Then, the necessary and sufficient condition for the finite-round LOCC to achieve globally optimal discrimination ( LOCC $_{\mathbb{N}}=\mathrm{GLOBAL}$ ) is determined by $\gamma_{-}$or $\gamma_{+}$:

$$
\begin{aligned}
& Y_{-}=\frac{\sin 2\left(\theta_{0}-\theta_{1}\right) \sqrt{\sin 2 \theta_{0}}+2 \sin \left(\theta_{0}-\theta_{1}\right) \sqrt{\sin 2 \theta_{1}}}{\left(\sin 2 \theta_{0}+\sin 2 \theta_{1}\right) \sqrt{\sin 2 \theta_{0}}}, \\
& Y_{+}=\frac{\sin 2\left(\theta_{0}+\theta_{1}\right) \sqrt{\sin 2 \theta_{0}}+2 \cos \left(\theta_{0}+\theta_{1}\right) \sqrt{\sin 2 \theta_{1}}}{\left(\sin 2 \theta_{0}+\sin 2 \theta_{1}\right) \sqrt{\sin 2 \theta_{0}}},
\end{aligned}
$$

where $\gamma_{-}$and $\gamma_{+}$are the ratios that correspond to the cases of $X=$ $\theta_{0}$ and $X=\frac{\pi}{2}-\theta_{0}$, respectively. That is, NLWE does not occur if $r$ is $\gamma_{-}$or $\gamma_{+} \cdot \gamma_{-}\left(\gamma_{+}\right)$becomes positive when $\theta_{1}<\theta_{0}\left(\theta_{1}<\frac{\pi}{2}-\theta_{0}\right)$. Therefore, for the mirror-symmetric states of Eq. (2), there is at least one set of nonzero prior probabilities in which NLWE does not occur. Note that when $\theta_{0} \neq \frac{\pi}{4}$, there is a finite gap $\mathcal{G}$ between $\gamma_{-}$and $\gamma_{+}$:

$\mathcal{G}=\left\{x \in \mathbb{R} \mid \gamma_{-}<x<\gamma_{+}\right\}$.

Until now, we have studied the possibility that a finite-round LOCC can achieve the global minimum-error probability of $\mathcal{E}$. Since an SEP can perform better than a finite-round LOCC in MD, now we will investigate a case that an SEP can obtain the global guessing probability $P_{\text {guess }}^{\mathrm{GLOBAL}}$ of $\mathcal{E}$. If we state the result in advance, as shown in Figure 1, when $r \notin \mathcal{G}$ (yellow), the global minimumerror probability of the quantum ensemble $\mathcal{E}$ can be achieved through an SEP. However, when $r \in \mathcal{G}$ (green), the global

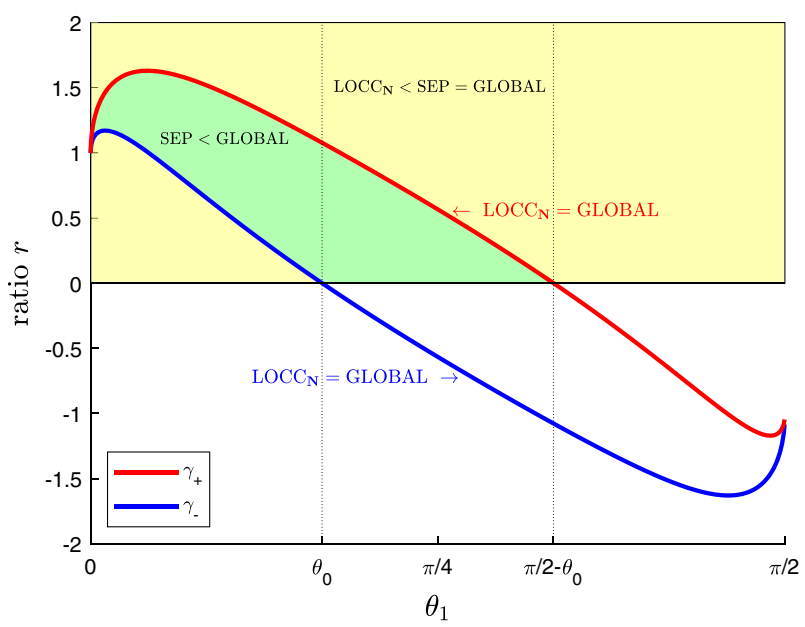

Fig. 1 Appearance of NLWE. When $\theta_{0} \neq \frac{\pi}{4}$, if $\theta_{1}<\theta_{0}$, both singular rates $\gamma_{-}$and $\gamma_{+}$are valid. However, when $\theta_{1} \geq \frac{\pi}{2}-\theta_{0}$, both $\gamma_{-}$and $\gamma_{+}$ are not valid. If $\theta_{0} \leq \theta_{1}<\frac{\pi}{2}-\theta_{0}, \gamma_{+}$is valid but $\gamma_{-}$is not. When the ratio $r$ becomes $\gamma_{-}$(blue) or $\gamma_{+}$(red), the global minimum-error probability of a quantum ensemble $\mathcal{E}$ can be achieved by a finiteround LOCC, otherwise it cannot be obtained by finite-round LOCC. Furthermore, if $r \notin \mathcal{G}$ (yellow), the global minimum-error probability of the quantum ensemble $\mathcal{E}$ can be achieved by means of a separable measurement. Meanwhile, when $r \in \mathcal{G}$ (green), the global minimum-error probability of a quantum state ensemble $\mathcal{E}$ cannot be obtained by a separable measurement. 


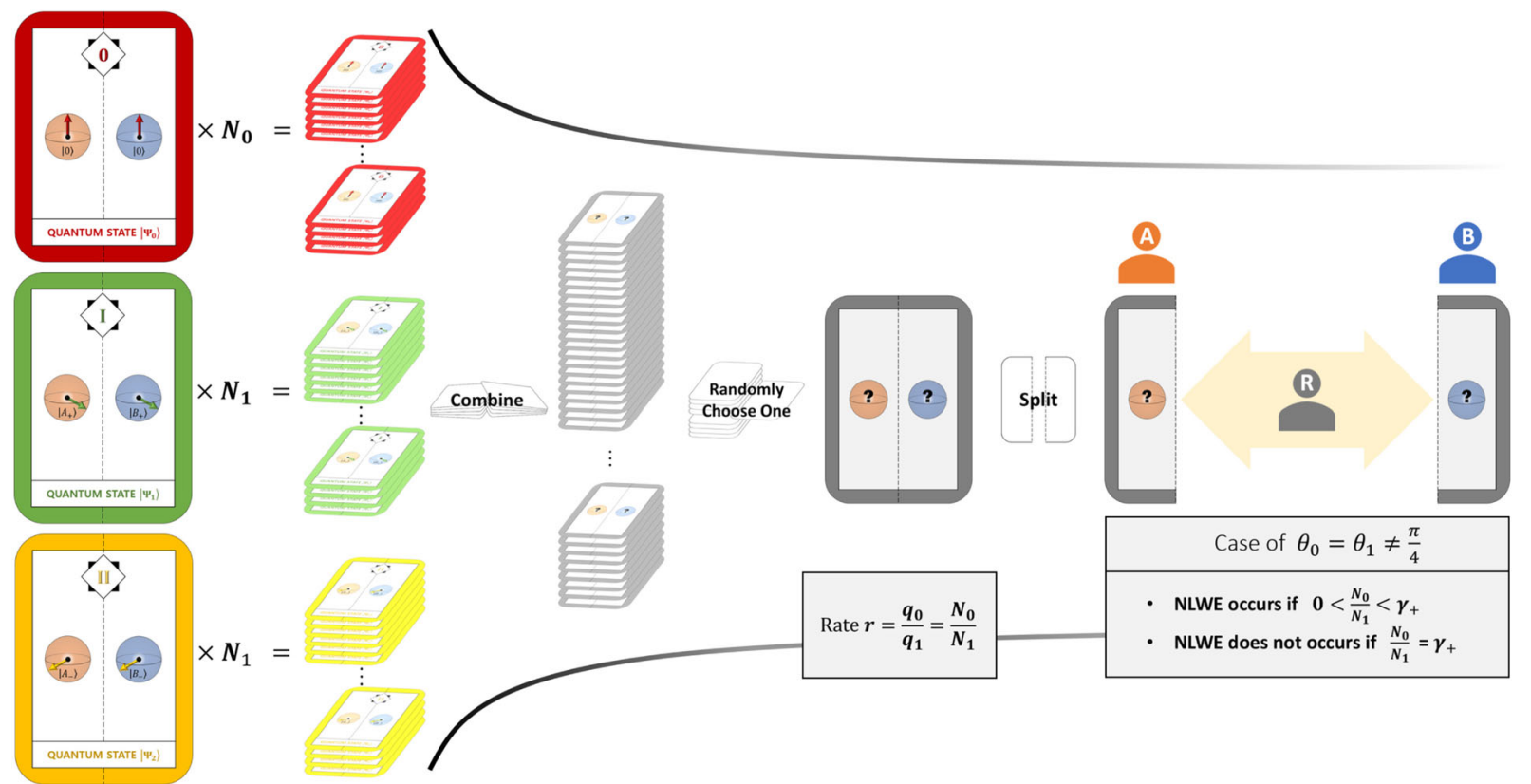

Fig. 2 NLWE in the case of $\boldsymbol{\theta}_{\mathbf{1}}=\boldsymbol{\theta}_{\mathbf{0}} \neq \frac{\boldsymbol{\pi}}{\mathbf{4}}$. In two-qubit system, a party $R$ prepares $N_{0}$ number of quantum state $\left|\Psi_{0}\right\rangle$ (red), $N_{1}$ number of quantum state $\left|\Psi_{1}\right\rangle$ (green), and $N_{1}$ number of quantum state $\left|\Psi_{2}\right\rangle$ (yellow). $R$ combines $N_{0}+2 N_{1}$ quantum systems and randomly chooses one out of them. Then, the prior probability $q_{1}$ of quantum state $\left|\Psi_{1}\right\rangle$ is equal to the prior probability $q_{2}$ of quantum state $\left|\Psi_{2}\right\rangle$. The ratio $r$ between the prior probability $q_{0}$ of quantum state $\left|\Psi_{0}\right\rangle$ and the prior probability $q_{1}$ is $N_{0} / N_{1}$. After the preparation, $R$ sends each qubit to $A$ and $B$, respectively. When $0<r<\gamma_{+}$is satisfied, NLWE occurs in the view of MD, but if $r=\gamma_{+}$, NLWE does not occur. More explicitly, because $\gamma_{+}$becomes $343 / 600$ when $\theta_{0}$ satisfies $\sin \theta_{0}=3 / 5$, if $N_{1}=600$, NLWE does not occur when $N_{0}$ is 343 . However, NLWE occurs in the case of $N_{0}=1,2, \ldots, 342$.

minimum-error probability of a quantum state ensemble $\mathcal{E}$ cannot be obtained by an SEP. This is obtained by applying the result of Duan et al. ${ }^{11}$ (see "Methods") to an orthonormal basis $\left\{\left|\Phi_{0}\right\rangle,\left|\Phi_{1}\right\rangle,\left|\Phi_{2}\right\rangle,\left|\Psi_{\theta_{0}}^{-}\right\rangle\right\}$; the necessary and sufficient condition that SEPs can perform the perfect discrimination of $\left\{\left|\Phi_{i}\right\rangle\left\langle\Phi_{i}\right|\right\}_{i=0}^{2}$ becomes $\sin 2 x \leq \sin 2 \theta_{0}$, which can be rewritten as $x \notin\left(\theta_{0}, \frac{\pi}{2}-\theta_{0}\right)$. For $0<x<\frac{\pi}{2}$, the rate $r$ strictly increases as a function of $X$. Therefore, $r \notin \mathcal{G}$ becomes the necessary and sufficient condition for $r$, when globally optimal discrimination of $\mathcal{E}$ can be achieved by an SEP: SEP $=$ GLOBAL. One obtains $r \in$ $\mathcal{G}$ if and only if the global minimum-error probability of $\mathcal{E}$ cannot be accomplished by an SEP: SEP $<$ GLOBAL. Therefore, two singular rates $\gamma_{-}$and $\gamma_{+}$form a boundary between SEP $=$GLOBAL and SEP $<$ GLOBAL. Figure 1 displays the behavior of two rates $\gamma_{-}$and $\gamma_{+}$when $\theta_{0} \neq \frac{\pi}{4}$. Because SEP $<$ GLOBAL includes $\overline{\mathrm{LOCC}}<\mathrm{GLOBAL}$, when $r$ belongs to $\mathcal{G}$, the NLWE occurs, i.e., $P_{\text {guess }}^{\overline{\mathrm{LOCC}}}<P_{\text {guess }}^{\mathrm{GLOBAL}}$. This means that, for the mirror-symmetric states of Eq. (2), there are infinite numbers of sets of nonzero prior probabilities revealing NLWE.

Our results speak of the following fact. When three product states $\left\{\left|\Psi_{i}\right\rangle\left\langle\Psi_{i}\right|\right\}_{i=0}^{2}$ are specified, corresponding to fixed $\theta_{1}$ and $\theta_{0}$, NLWE depends on nonzero prior probabilities. For the mirrorsymmetric states of Eq. (2), there are infinite numbers of sets of nonzero prior probabilities where NLWE occurs (SEP < GLOBAL) and there is at least one set of nonzero prior probabilities in which NLWE does not occur $\left(\right.$ LOCC $_{\mathbb{N}}=$ GLOBAL). It tells that those different ensembles, which correspond to different prior probabilities, although composed of the same separable quantum states, exhibit different behaviors in terms of nonlocality. The following theorem summarizes the result.

Theorem 1 For the MD of nonorthogonal states given as Eq. (2), the NLWE explicitly depends on the prior probabilities.
For example, let us consider the case of $\theta_{1}=\theta_{0} \neq \frac{\pi}{4}$. Then, $\left|A_{ \pm}\right\rangle$ and $\left|B_{ \pm}\right\rangle$consisting of $\left|\Psi_{1}\right\rangle$ and $\left|\Psi_{2}\right\rangle$ become

$$
\left|A_{ \pm}\right\rangle=\cos \theta_{0}|0\rangle \pm \sin \theta_{0}|1\rangle \text { and }\left|B_{ \pm}\right\rangle=\frac{1}{\sqrt{2}}(|0\rangle \pm|1\rangle) \text {. }
$$

In this case, since $\gamma_{-}$is zero, the necessary and sufficient condition of $\mathrm{SEP}<\mathrm{GLOBAL}$ is

$0<r<\gamma_{+}=\cos 2 \theta_{0}+\frac{\cos 2 \theta_{0}}{\sin 2 \theta_{0}}$

and the one of LOCC $_{\mathbb{N}}=$ GLOBAL is $r=\gamma_{+}$. Therefore, NLWE occurs if $r<\gamma_{+}$but not if $r=\gamma_{+}$. Figure 2 illustrates in detail how NLWE can occur by different mixing of quantum states in the case of $\theta_{1}=\theta_{0} \neq \frac{\pi}{4}$.

\section{Case where identical quantum states are shared at different locations}

Now, we are ready to investigate the case where identical quantum states are shared in different locations. It corresponds to the case when $\theta_{0}=\frac{\pi}{4}$ in Eq. (2). We will prove that even in this case, NLWE occurs and depends on the prior probability of the quantum ensemble. In this case, $\left\{\left|\Psi_{i}\right\rangle\right\}_{i=0}^{2}$ can be rewritten as (the range of $\epsilon$ becomes $\left.0<\epsilon<\frac{\pi}{4}\right)$ :

$$
\begin{aligned}
\left|\Psi_{0}\right\rangle & =|00\rangle \\
\left|\Psi_{1}\right\rangle & =\left|D_{+}\right\rangle \otimes\left|D_{+}\right\rangle \\
& =\cos ^{2} \epsilon|00\rangle+\sin ^{2} \epsilon|11\rangle+\sqrt{2} \cos \epsilon \sin \epsilon\left|\Psi_{\frac{\pi}{4}}^{+}\right\rangle \\
\left|\Psi_{2}\right\rangle & =\left|D_{-}\right\rangle \otimes\left|D_{-}\right\rangle \\
& =\cos ^{2} \epsilon|00\rangle+\sin ^{2} \epsilon|11\rangle-\sqrt{2} \cos \epsilon \sin \epsilon\left|\Psi_{\frac{\pi}{4}}^{+}\right\rangle
\end{aligned}
$$

where

$$
\left|D_{ \pm}\right\rangle=\cos \epsilon|0\rangle \pm \sin \epsilon|1\rangle \text {. }
$$




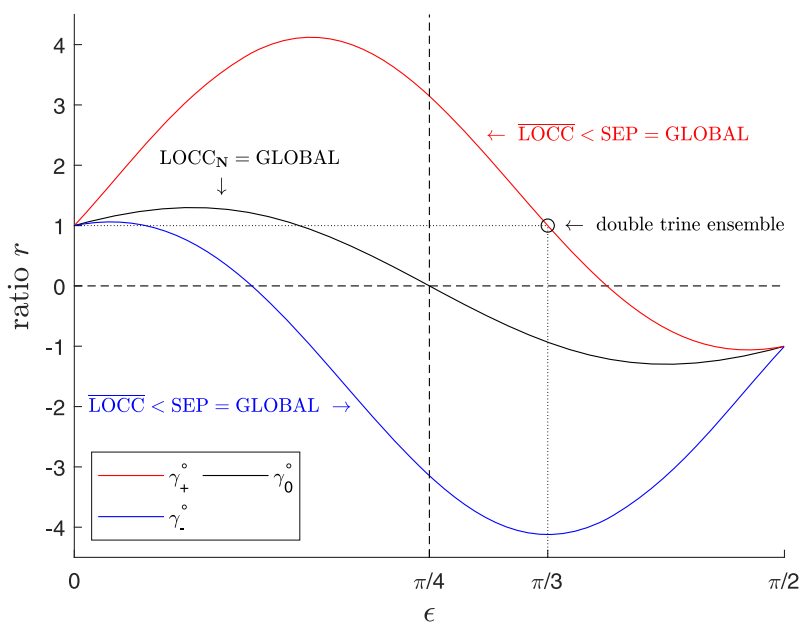

Fig. 3 Appearance of NLWE in a singular case. $\gamma_{0}^{\circ}$ (black), $\gamma_{+}^{0}$ (red), and $\gamma_{-}^{0}$ (blue) corresponding to $\epsilon \in\left(0, \frac{\pi}{2}\right)$ : when $0<\epsilon<\frac{\pi}{4}, \gamma_{0}^{\circ}$ and $\gamma_{+}^{\circ}$ are positive. However, when $\frac{\pi}{4} \leq \epsilon<\frac{\pi}{2}, \gamma_{0}^{\circ}$ and $\gamma_{-}^{\circ}$ are non-positive. Because the ratio $r$ must be positive, when $0<\epsilon<\frac{\pi}{4}$, there are two cases when NLWE occurs and does not. However, when $\frac{\pi}{4} \leq \epsilon<\frac{\pi}{2}$, it is impossible to explicitly indicate the occurrence of NLWE. The double trine ensemble corresponds to the case of $\epsilon=\frac{\pi}{3}$ and $r=1$.

For the quantum ensemble given as $\mathcal{E}=\left\{q_{i}, \rho_{i}\right\}_{i=0}^{2}$ consisting of above two-qubit product states $\rho_{i}=\left|\Psi_{i}\right\rangle\left\langle\Psi_{i}\right|$ with prior probabilities $q_{i}$, one can find the optimal measurement vectors $\left\{\left|\Phi_{i}\right\rangle\right\}_{i=0}^{2}$, which corresponds to the optimal measurement of Eq. (3) at $\theta_{0}=\frac{\pi}{4}$. The optimal measurement factor $x \in\left(0, \frac{\pi}{2}\right)$ satisfies the following relation:

$$
\begin{aligned}
r= & \frac{1}{\sin 2 X}\left[\sin 2 X \cos 2 \epsilon-\frac{1}{2} \cos 2 X \sin ^{2} 2 \epsilon\right. \\
& \left.+\sqrt{2} \sin 2 \epsilon\left(\sin X \cos ^{2} \epsilon-\cos X \sin ^{2} \epsilon\right)\right] .
\end{aligned}
$$

By the similar analysis as before, one can see that $\left|\Phi_{1}\right\rangle$ and $\left|\Phi_{2}\right\rangle$ are entangled except in the case of $X=\frac{\pi}{4}$. In other words, one can obtain $X=\frac{\pi}{4}$ if and only if at least two of $\left\{\left|\Phi_{i}\right\rangle\left\langle\Phi_{i}\right|\right\}_{i=0}^{2}$ are product states. If one applies $X=\frac{\pi}{4}$ to the right-hand side of (10), the ratio $r$ can be expressed as $\gamma_{0}^{\circ}$ :

$\gamma_{0}^{\circ}=\cos 2 \epsilon(1+\sin 2 \epsilon)$.

That is, $X$ becomes $\frac{\pi}{4}$ if and only if $r=\gamma_{0}^{\circ}$. It should be noted that when $\frac{\pi}{4} \leq \epsilon<\frac{\pi}{2}, r=\gamma_{0}^{\circ}$ is not satisfied because $\gamma_{0}^{\circ}$ is non-positive. Therefore, one has $r=\gamma_{0}^{\circ}$ if and only if the global minimum-error probability of $\left\{q_{i},\left|\Psi_{i}\right\rangle\left\langle\Psi_{i}\right|\right\}_{i=0}^{2}$ can be achieved by a finite-round LOCC: $\operatorname{LOCC}_{\mathbb{N}}=$ GLOBAL. This means that, for the mirrorsymmetric states of Eq. (9), there is at least one set of nonzero prior probabilities where NLWE does not occur.

In order to find a case where an asymptotic LOCC cannot provide the global minimum-error probability, one may consult the result by Chitambar and Hsieh ${ }^{15}$, which states that $\left\{\left|\Phi_{i}\right\rangle\left\langle\Phi_{i}\right|\right\}_{i=0}^{2}$ of the double trine ensemble $\left(\epsilon=\frac{\pi}{3}, r=1\right)$ cannot fulfill the necessary condition of perfect asymptotic LOCC discrimination ${ }^{21}$. Therefore, for all quantum ensemble $\mathcal{E}$ with $\left\{\left|\Phi_{i}\right\rangle\left\langle\Phi_{i}\right|\right\}_{i=0}^{2}$ corresponding to $(\epsilon, r)=\left(\frac{\pi}{3}, 1\right)$, an asymptotic LOCC cannot provide the global guessing probability, i.e., $P_{\text {guess }}^{\overline{\mathrm{LOCC}}}<P_{\text {guess }}^{\mathrm{GLOBAL}}$. Because the value of $X$, derived from Eq. (10) with $r=1$ and $\epsilon=\frac{\pi}{3}$, satisfies $\sin X=(\sqrt{2}+1) / \sqrt{6},\left\{\left|\Lambda_{i}^{+}\right\rangle\left\langle\Lambda_{i}^{+}\right|\right\}_{i=0}^{2}$ cannot be perfectly discriminated by asymptotic LOCC, where

$$
\begin{aligned}
& \left|\Lambda_{0}^{ \pm}\right\rangle=\frac{\sqrt{2} \pm 1}{\sqrt{6}}|00\rangle-\frac{\sqrt{2} \mp 1}{\sqrt{6}}|11\rangle, \\
& \left|\Lambda_{1}^{ \pm}\right\rangle=\frac{1}{\sqrt{2}}\left(\frac{\sqrt{2} \mp 1}{\sqrt{6}}|00\rangle+\frac{\sqrt{2} \pm 1}{\sqrt{6}}|11\rangle\right)+\frac{1}{\sqrt{2}}\left|\Psi_{\frac{\pi}{4}}^{+}\right\rangle, \\
& \left|\Lambda_{2}^{ \pm}\right\rangle=\frac{1}{\sqrt{2}}\left(\frac{\sqrt{2} \mp 1}{\sqrt{6}}|00\rangle+\frac{\sqrt{2} \pm 1}{\sqrt{6}}|11\rangle\right)-\frac{1}{\sqrt{2}}\left|\Psi_{\frac{\pi}{4}}^{+}\right\rangle .
\end{aligned}
$$

In addition, $\left\{\left|\Lambda_{i}^{-}\right\rangle\left\langle\Lambda_{i}^{-}\right|\right\}_{i=0}^{2}$ cannot be perfectly discriminated by asymptotic LOCC because $\left|\Lambda_{i}^{+}\right\rangle\left\langle\Lambda_{i}^{+}\right|$and $\left|\Lambda_{i}^{-}\right\rangle\left\langle\Lambda_{i}^{-}\right|$can be exchanged by the local unitary operation $\sigma_{X} \otimes \sigma_{X}$, where $\sigma_{X}=|0\rangle\langle 1|+| 1\rangle\langle 0|$. One should note that $\left|\Lambda_{i}^{ \pm}\right\rangle$is $\left|\Phi_{i}\right\rangle$ with $X_{r}$ which corresponds to $\sin X=(\sqrt{2} \pm 1) / \sqrt{6}$. In both of these cases, $X$ satisfies $\sin X=1 / 3$. Therefore, $\left\{\left|\Phi_{i}\right\rangle\left\langle\Phi_{i}\right|\right\}_{i=0}^{2}$, when $\sin X=1 / 3$, cannot be perfectly discriminated by asymptotic LOCC. If one applies $\sin X=(\sqrt{2} \pm 1) / \sqrt{6}$ to the right-hand side of Eq. (10), the ratio $r$ can be expressed as $\gamma_{ \pm}^{\circ}$ :

$Y_{ \pm}^{\circ}=\cos 2 \epsilon(\sqrt{6} \sin 2 \epsilon+1) \pm \sin 2 \epsilon(\sqrt{2} \sin 2 \epsilon+\sqrt{3})$.

Therefore, if $r=\gamma_{+}^{\circ}$ or $\gamma_{-}^{\circ}$, the global minimum-error probability of $\left\{q_{i},\left|\Psi_{i}\right\rangle\left\langle\Psi_{i}\right|\right\}_{i=0}^{2}$ cannot be achieved by asymptotic LOCC: $\overline{\text { LOCC }}<$ GLOBAL. This implies that, for the mirror-symmetric states of Eq. (9), there is at least one set of nonzero prior probabilities in which NLWE occurs.

These results imply the following fact: when three product states are specified that correspond to a fixed $\epsilon$, NLWE depends on nonzero prior probabilities. More explicitly, for the mirrorsymmetric states of Eq. (9), there is at least one set of nonzero prior probabilities where NLWE occurs ( $\overline{\mathrm{LOCC}}<\mathrm{GLOBAL}$ ), and there is a set of nonzero prior probabilities in which NLWE does not occur $\left(\right.$ LOCC $\left._{\mathbb{N}}=\mathrm{GLOBAL}\right)$. The situation is illustrated in Fig, 3, which displays the rates $\gamma_{0}^{\circ}$ and $\gamma_{+}^{\circ}$ corresponding to $\epsilon$. For the curve $\gamma_{0}^{\circ}$ shown in Fig. 3 , there is no NLWE, but in the other two curves, $\gamma_{+}^{\circ}$ and $\gamma_{-}^{\circ}$, NLWE occurs. That is, when $\epsilon<\frac{\pi}{4}$, NLWE in MD depends on the prior probability, in contrast to the perfect discrimination of orthogonal states. The following theorem summarizes the result.

Theorem 2 For the MD of nonorthogonal states given as Eq. (9), the NLWE explicitly depends on the prior probabilities.

\section{DISCUSSION}

In this study, we showed that nonlocality depends on not only separable quantum states of a quantum ensemble but also the prior probabilities of given quantum states. It was proved by showing that nonlocal measurement on the mirror-symmetric quantum ensemble provides more information about the unentangled states than LOCC does when the prior probabilities have special values. Our result implies that nonlocality can be revealed by way of mixing given separable quantum states. In the view that NLWE of orthogonal states does not show explicit dependence on prior probabilities, our result is surprising.

Also, we considered the specific quantum states with a mirror symmetry, which are used to prove that NLWE depends explicitly on the prior probability of the quantum ensemble. We should note that the quantum states are parametrized by variables $\theta_{0}$ and $\theta_{1}$, and there are infinite numbers of quantum states that reveal NLWE.

Here, we provided a new type of quantum ensemble with mirror symmetry. Moreover, by exploiting it, we can provide cases where NLWE can be shown. However, because there are cases where one cannot decide on the occurrence of NLWE, more investigation is needed. Further, it is interesting to know how frequently NLWE can occur. The weakening of mirror symmetry may help to accomplish the investigation. 
In our work, we provided an actual result on the explicit dependence of prior probability in the behavior of NLWE. From this point of view, the following needs to be noted. In the perfect discrimination of nine orthogonal states of ref. ${ }^{3}$, NLWE occurs regardless of prior probability. Furthermore, since finite-round LOCC can perfectly discriminate pure orthogonal product states (for instance, two multipartite states ${ }^{23}$, three $2 \otimes 2$ states, and four $2 \otimes 2$ states $^{5}$ ), NLWE does not occur regardless of prior probability. In perfect discrimination of orthogonal multipartite states, measurements discriminating perfectly the quantum states are independent of prior probabilities. Therefore, in the cases, NLWE depends only on those quantum states, not on the values of nonzero prior probabilities. It implies that in perfect discrimination of orthogonal multipartite states, one cannot see explicit dependence on prior probabilities. However, in MED of nonorthogonal states, optimal measurements may depend on nonzero prior probabilities. Therefore, in this case, NLWE may rely not only on quantum states but also on prior probabilities. It should be noted that in two bipartite nonorthogonal pure states, LOCC can provide optimal discrimination regardless of prior probabilities ${ }^{24}$. Therefore, the discrimination of nonorthogonal states does not guarantee the explicit dependence of prior probabilities. It is unknown whether nonorthogonal quantum states can provide NLWE regardless of prior probability in MED. We leave it an open problem. If such a set of nonorthogonal states exists, one can classify NLWE of nonorthogonal states by explicit dependence of prior probabilities.

Now, let us discuss some implications of our results. Our results tell that according to the quantum states in our paper, local optimal discrimination depends explicitly on the variation of prior probability. Since orthogonal states (i.e., local distinguishable states), which can be discriminated by finite-round LOCC, can be applied to quantum data hiding ${ }^{28-31}$ and quantum secret sharing $^{32-34}$, our result may be used in extending the result of orthogonal states to the application of nonorthogonal states.

\section{METHODS}

\section{Mirror-symmetric ensemble}

Let us consider quantum state ensemble $\mathcal{E}=\left\{q_{i}, \rho_{i}\right\}_{i=0}^{2}$ consisting of linearly independent pure states $\rho_{i}=\left|\psi_{i}\right\rangle\left\langle\psi_{i}\right|$ such that

$$
\begin{aligned}
& \left|\psi_{0}\right\rangle=\sin a\left|E_{0}\right\rangle+\cos a\left|E_{1}\right\rangle, \\
& \left|\psi_{1}\right\rangle=\sin \beta\left|E_{1}\right\rangle+\cos \beta\left|E_{2}\right\rangle, \\
& \left|\psi_{2}\right\rangle=\sin \beta\left|E_{1}\right\rangle-\cos \beta\left|E_{2}\right\rangle, 0<\alpha, \beta<\frac{\pi}{2} .
\end{aligned}
$$

In addition, the prior probabilities $q_{0}, q_{1}$, and $q_{2}$ are supposed to be

$\frac{q_{0}}{q_{1}}=\frac{q_{0}}{q_{2}}=r$ for some $r \in(0, \infty)$.

$\left\{\left|E_{i}\right\rangle\right\}_{i=0}^{2}$ is an orthonormal basis of three-dimensional complex Hilbert space $\mathcal{H}$. Note that $\rho_{0} \not \rho_{1}$ and $\rho_{0} \not \perp \rho_{2}$, where $\not$ indicates nonorthogonality. If $U$ is a unitary operator $\left|E_{0}\right\rangle\left\langle E_{0}|+| E_{1}\right\rangle\left\langle E_{1}|-| E_{2}\right\rangle\left\langle E_{2}\right|$, the ensemble $\mathcal{E}$ satisfies the following property:

$U q_{0} \rho_{0} U^{\dagger}=q_{0} \rho_{0}, U q_{1} \rho_{1} U^{\dagger}=q_{2} \rho_{2}, U q_{2} \rho_{2} U^{\dagger}=q_{1} \rho_{1}$.

Conversely, when $\left\{q_{i}, \rho_{i}\right\}_{i=0}^{2}$ is a quantum ensemble consisting of three linearly independent pure states with $\rho_{0} \not \rho_{1}$ and $\rho_{0} \not \rho_{2}$, if there exists a unitary operator $U$ satisfying (14), then the ensemble can be expressed as (12) and (13). Therefore, one can tell that the ensemble $\mathcal{E}$ has mirror symmetry.

Until now, the general solution of MD of quantum state ensemble $\mathcal{E}$ was unknown. However, a few important results ${ }^{35}$ was found. First, the minimum-error measurement (the optimal POVM on $\mathcal{H})\left\{M_{i}\right\}_{i=0}^{2}$ is unique and has a form of a projective measurement composed of orthonormal vectors $\left|\phi_{i}\right\rangle$, which can be expressed by $M_{i}=\left|\phi_{i}\right\rangle\left\langle\phi_{i}\right|$. Secondly, for each $i$, $\left|\psi_{i}\right\rangle$ and $\left|\phi_{i}\right\rangle$ are not perpendicular, $\left\langle\psi_{i} \mid \phi_{i}\right\rangle \neq 0$. From these properties, one can prove that the optimal measurement vectors $\left|\phi_{i}\right\rangle$ for $\mathcal{E}$ can be expressed as

$$
\begin{aligned}
& \left|\phi_{0}\right\rangle=\sin X\left|F_{0}\right\rangle-\cos X\left|F_{1}\right\rangle, \\
& \left|\phi_{1}\right\rangle=\frac{1}{\sqrt{2}}\left(\cos X\left|F_{0}\right\rangle+\sin X\left|F_{1}\right\rangle\right)+\frac{1}{\sqrt{2}}\left|F_{2}\right\rangle, \\
& \left|\phi_{2}\right\rangle=\frac{1}{\sqrt{2}}\left(\cos X\left|F_{0}\right\rangle+\sin X\left|F_{1}\right\rangle\right)-\frac{1}{\sqrt{2}}\left|F_{2}\right\rangle,
\end{aligned}
$$

where

$$
\begin{aligned}
& \left|F_{0}\right\rangle=\cos a\left|E_{1}\right\rangle+\sin a\left|E_{0}\right\rangle, \\
& \left|F_{1}\right\rangle=\sin a\left|E_{1}\right\rangle-\cos a\left|E_{0}\right\rangle,\left|F_{2}\right\rangle=\left|E_{2}\right\rangle .
\end{aligned}
$$

Here, we use the optimal measurement's symmetry, derived from the mirror symmetry of a given ensemble. $X$ is the value which is determined by applying the necessary condition for the optimal measurement $M_{0}\left(q_{0} \rho_{0}-q_{1} \rho_{1}\right) M_{1}=0$ (in our case, it corresponds to the condition of $\left.\left.r\left\langle\phi_{0} \mid \psi_{0}\right\rangle\left\langle\psi_{0} \mid \phi_{1}\right\rangle=\left\langle\phi_{0} \mid \psi_{1}\right\rangle\left\langle\psi_{1} \mid \phi_{1}\right\rangle\right)^{18,19,36}\right)$ to Eq. (15). It becomes $\theta \in\left(a, \frac{\pi}{2}\right)$, which satisfies the following equation:

$r \sin 2 \theta=\sin ^{2} \beta \sin 2(\theta-a)+\sin 2 \beta \sin (\theta-a)$.

If one substitutes $\sin (\theta-a)$ into $\lambda$, then Eq. (17) is expressed by the following non-trivial fourth-order equation, and $X$ has a very complex form,

$$
\begin{aligned}
& 4\left(r^{2}-2 r \cos 2 a \sin ^{2} \beta+\sin ^{4} \beta\right) \lambda^{4}+4 r \sin 2 a \sin 2 \beta \lambda^{3} \\
& \quad-4\left(r^{2}-2 r \cos 2 a \sin ^{2} \beta-\sin ^{2} \beta \cos 2 \beta\right) \lambda^{2} \\
& \quad-2 r \sin 2 a \sin 2 \beta \lambda+r^{2} \sin ^{2} 2 a=0 .
\end{aligned}
$$

When $a, \beta$, and $r$ are given, finding $X$ is very complicated. However, if $x$ is given, finding $\alpha, \beta$, and $r$, corresponding to $x$, is not difficult. This is because the constraint on $r$, which is the ratio between the prior probabilities $q_{0}$ and $q_{1}\left(\right.$ or $q_{2}$ ), requires only positiveness (i.e., $\left.0<r<\infty\right)$. Therefore, when an arbitrary $X$ is given, if $r$ satisfies the following condition, then (15) are the optimal measurement vectors,

$r=\frac{\sin ^{2} \beta \sin 2(x-a)+\sin 2 \beta \sin (x-a)}{\sin 2 x}$.

Note that (18) is derived from (17).

\section{Necessary and sufficient condition for global minimum-error probability}

Recently, Chitambar and Hsieh provided an important result ${ }^{15}$. The result states that MD of $n$ nonorthogonal linearly independent pure states can be reduced to the perfect discrimination problem of $n$ orthogonal pure states. Specifically, it was shown that when $\left\{q_{i},\left|\psi_{i}\right\rangle\left\langle\psi_{i}\right|\right\}_{i=0}^{n-1}$ is a quantum ensemble of $n$ nonorthogonal linearly independent pure states, a measurement provides the guessing probability if and only if the measurement can perfectly discriminate $n$ orthogonal pure states $\left\{\left|\phi_{i}\right\rangle\left\langle\phi_{i}\right|\right\}_{i=0}^{n-1}$. Here, $\left\{\left|\phi_{i}\right\rangle\right\}_{i=0}^{n-1}$ is an orthonormal basis of $\mathcal{H}$, where $\mathcal{H}$ is the $n$-dimensional Hilbert space generated by $\left\{\left|\psi_{i}\right\rangle\right\}_{i=0}^{n-1}$. This basis has the following property: $\left\{\left|\phi_{i}\right\rangle\left\langle\phi_{i}\right|\right\}_{i=0}^{n-1}$ is the unique optimal POVM of $\left\{q_{i},\left|\psi_{i}\right\rangle\left\langle\psi_{i}\right|\right\}_{i=0}^{n-1}$ on $\mathcal{H}$. It should be noted that when the space, in which quantum states and measurement operators are defined, is larger than $\mathcal{H}$, the minimum-error measurement of $\left\{q_{i},\left|\psi_{i}\right\rangle\left\langle\psi_{i}\right|\right\}_{i=0}^{n-1}$ is not unique. Therefore, in the case of a multi-party system, the global minimum-error probability of $\left\{q_{i},\left|\psi_{i}\right\rangle\left\langle\psi_{i}\right|\right\}_{i=0}^{n-1}$ can be achieved by finite-round (asymptotic) LOCC if and only if $\left\{\left|\phi_{i}\right\rangle\left\langle\phi_{i}\right|\right\}_{i=0}^{n-1}$ can be perfectly discriminated using the finite-round (asymptotic) LOCC.

\section{Perfect discrimination of quantum states by SEPs}

Duan et al. ${ }^{11}$ tells that in a two-qubit system, when $\left\{\left|\bar{\Xi}_{0}\right\rangle,\left|\bar{\Xi}_{1}\right\rangle,\left|\bar{\Xi}_{2}\right\rangle,|\Gamma\rangle\right\}$ is an orthonormal basis, the necessary and sufficient condition for $\left\{\left|\bar{\Xi}_{i}\right\rangle\left\langle\bar{\Xi}_{i}\right|\right\}_{i=0}^{2}$ to be distinguished by a SEP is as follows: (i) $\bar{E}_{k} \Gamma^{-1}$ has two antiparallel eigenvalues for each entangled $\left|\Xi_{k}\right\rangle$ and (ii) $\sum_{i=0}^{2} C\left(\Xi_{i}\right)=C(\Gamma)$, where $\Psi$ corresponding to $|\Psi\rangle$ is a $2 \times 2$ matrix satisfying $|\Psi\rangle=(I \otimes$ $\Psi)\left|\Phi_{\frac{\pi}{4}}^{+}\right\rangle$and $C(\Psi)$ denotes the concurrence of $|\Psi\rangle$, i.e., $C(\Psi)=|\operatorname{det} \Psi|$. In particular, when $|\Gamma\rangle$ is maximally entangled, $\left\{\left|\bar{E}_{i}\right\rangle\left\langle\bar{\Xi}_{i}\right|\right\}_{i=0}^{2}$ can be perfectly discriminated by an SEP if and only if $\sum_{i=0}^{2} C\left(\bar{\Xi}_{i}\right)=1$. 


\section{DATA AVAILABILITY}

All data needed to evaluate the conclusions in the paper are present in the paper and/or the Supplementary Information.

Received: 27 August 2020; Accepted: 17 April 2021;

Published online: 25 May 2021

\section{REFERENCES}

1. Peres, A. \& Wootters, W. K. Optimal detection of quantum information. Phys. Rev. Lett. 66, 1119 (1991).

2. Horodecki, R., Horodecki, P., Horodecki, M. \& Horodecki, K. Quantum entanglement. Rev. Mod. Phys. 81, 865 (2009).

3. Bennett, C. H. et al. Quantum nonlocality without entanglement. Phys. Rev. A 59, 1070 (1999).

4. Ghosh, S., Kar, G., Roy, A., Sen(De), A. \& Sen, U. Distinguishability of Bell states. Phys. Rev. Lett. 87, 277902 (2001).

5. Walgate, J. \& Hardy, L. Nonlocality, asymmetry, and distinguishing bipartite states. Phys. Rev. Lett. 89, 147901 (2002).

6. Ghosh, S., Kar, G., Roy, A. \& Sarkar, D. Distinguishability of maximally entangled states. Phys. Rev. A 70, 022304 (2004).

7. Fan, H. Distinguishability and indistinguishability by local operations and classical communication. Phys. Rev. Lett. 92, 177905 (2004).

8. Watrous, J. Bipartite subspaces having no bases distinguishable by local operations and classical communication. Phys. Rev. Lett. 95, 080505 (2005).

9. Cohen, S. M. Local distinguishability with preservation of entanglement. Phys. Rev. A 75, 052313 (2007).

10. Duan, R., Feng, Y., Ji, Z. \& Ying, M. Distinguishing arbitrary multipartite basis unambiguously using local operations and classical communication. Phys. Rev. Lett. 98, 230502 (2007).

11. Duan, R., Feng, Y., Xin, Y. \& Ying, M. Distinguishability of quantum states by separable operations. IEEE Trans. Inf. Theory 55, 1320 (2009).

12. Bandyopadhyay, S. \& Walgate, J. Local distinguishability of any three quantum states. J. Phys. A 42, 072002 (2009).

13. Bandyopadhyay, S. More nonlocality with less purity. Phys. Rev. Lett. 106, 210402 (2011).

14. Bandyopadhyay, S. Entanglement, mixedness, and perfect local discrimination of orthogonal quantum states. Phys. Rev. A 85, 042319 (2012).

15. Chitambar, E. \& Hsieh, M. Revisiting the optimal detection of quantum information. Phys. Rev. A 88, 020302(R) (2013).

16. Chitambar, E., Leung, D., Mančinska, L., Ozols, M. \& Winter, A. Everything you always wanted to know about LOCC (but were afraid to ask). Commun. Math. Phys. 328, 303 (2014).

17. Helstrom, C. W. Quantum Detection and Estimation Theory (Academic, 1976).

18. Holevo, A. S. Probabilistic and Statistical Aspects of Quantum Thoery (North-Holland, 1979).

19. Yuen, H. P., Kennedy, R. S. \& Lax, M. Optimum testing of multiple hypotheses in Quantum Detection Theory. IEEE Trans. Inf. Theory 21, 125 (1975).

20. Ha, D. \& Kwon, Y. Discriminating $N$-qudit states using geometric structure. Phys. Rev. A 90, 022330 (2014).

21. Kleinmann, M., Kampermann, H. \& Bruß, D. Asymptotically perfect discrimination in the local-operation-and-classical-communication paradigm. Phys. Rev. A 84, 042326 (2011)

22. Childs, A. M., Leung, D., Mančinska, L. \& Ozols, M. A framework for bounding nonlocality of state discrimination. Commun. Math. Phys. 323, 1121 (2013).

23. Walgate, J., Short, A. J., Hardy, L. \& Vedral, V. Local distinguishability of multipartite orthogonal quantum states. Phys. Rev. Lett. 85, 4972 (2000).

24. Virmani, S., Sacchi, M. F., Plenioa, M. B. \& Markham, D. Optimal local discrimination of two multipartite pure states. Phys. Lett. A 288, 62 (2001).

25. Halder, S., Banik, M., Agrawal, S. \& Bandyopadhyay, S. Strong quantum nonlocality without entanglement. Phys. Rev. Lett. 122, 040403 (2019).

26. Bhattacharya, S. S., Saha, S., Guha, T. \& Banik, M. Nonlocality without entanglement: quantum theory and beyond. Phys. Rev. Res. 2, 012068(R) (2020).

27. Wootters, W. K. Entanglement of formation of an arbitrary state of two qubits. Phys. Rev. Lett. 80, 2245 (1998).

28. Terhal, B. M., DiVincenzo, D. P. \& Leung, D. W. Hiding bits in Bell states. Phys. Rev. Lett. 86, 5807 (2001).
29. DiVincenzo, D. P., Leung, D. W. \& Terhal, B. M. Quantum data hiding. IEEE Trans. Inf. Theory 48, 580 (2002).

30. Eggeling, T. \& Werner, R. F. Hiding classical data in multipartite quantum states. Phys. Rev. Lett. 89, 097905 (2002).

31. Matthews, W., Wehner, S. \& Winter, A. Distinguishability of quantum states under restricted families of measurements with an application to quantum data hiding. Commun. Math. Phys. 291, 813 (2009).

32. Markham, D. \& Sanders, B. C. Graph states for quantum secret sharing. Phys. Rev. A 78, 042309 (2008).

33. Rahaman, R. \& Parker, M. G. Quantum scheme for secret sharing based on local distinguishability. Phys. Rev. A 91, 022330 (2015).

34. Wang, J., Li, L., Peng, H. \& Yang, Y. Quantum-secret-sharing scheme based on local distinguishability of orthogonal multiqudit entangled states. Phys. Rev. A 95, 022320 (2017).

35. Eldar, Y. C. von Neumann measurement is optimal for detecting linearly independent mixed quantum states. Phys. Rev. A 68, 052303 (2003).

36. Barnett, S. M. \& Croke, S. On the conditions for discrimination between quantum states with minimum error. J. Phys. A 42, 062001 (2009).

\section{ACKNOWLEDGEMENTS}

We thank Jihwan Kim for his contribution to the early stage of this work. This work is supported by the Basic Science Research Program through the National Research Foundation of Korea (NRF) funded by the Ministry of Education, Science and Technology (NRF2018R1D1A1B07049420) and Institute of Information and Communications Technology Planning and Evaluation (IITP) grant funded by the Korean government (MSIT) (No. 2020001343, Artificial Intelligence Convergence Research Center (Hanyang University ERICA)). D.H. acknowledges support from the National Research Foundation of Korea (NRF) grant funded by the Korean government (Ministry of Science and ICT) (NRF2020M3E4A1080088).

\section{AUTHOR CONTRIBUTIONS}

D.H. and Y.K. contributed to design the ideas, perform the calculations, analyze the results, and write the manuscript.

\section{COMPETING INTERESTS}

The authors declare no competing interests.

\section{ADDITIONAL INFORMATION}

Correspondence and requests for materials should be addressed to D.H. or Y.K.

Reprints and permission information is available at http://www.nature.com/ reprints

Publisher's note Springer Nature remains neutral with regard to jurisdictional claims in published maps and institutional affiliations.

(i) Open Access This article is licensed under a Creative Commons adaptation, distribution and reproduction in any medium or format, as long as you give appropriate credit to the original author(s) and the source, provide a link to the Creative Commons license, and indicate if changes were made. The images or other third party material in this article are included in the article's Creative Commons license, unless indicated otherwise in a credit line to the material. If material is not included in the article's Creative Commons license and your intended use is not permitted by statutory regulation or exceeds the permitted use, you will need to obtain permission directly from the copyright holder. To view a copy of this license, visit http://creativecommons. org/licenses/by/4.0/.

(c) The Author(s) 2021, corrected publication 2021 